\title{
EVALUATION OF THE EMOTIONAL COMPONENT OF TRANSFORMABLE CLOTHING WITH SEMANTIC DIFFERENTIAL
}

\author{
Oksana Zakharkevich, Svetlana Kuleshova, Juliya Vovk \& Galina Khimich \\ Khmelnitskyi National University \\ Institytska str., 11, 29016 Khmelnitskyi, Ukraine \\ phone: 38067-749-16-47, e-mail: zbir_vukladach@ukr.net
}

\begin{abstract}
This paper aims to evaluate the emotional component of the transformable clothing by using semantic differential. The process of evaluating consumer's emotional experience consists of steps that provide an ability to classify the Kansei attributes of transformable clothes. The results of evaluation are to be used as a data set to fill the database of knowledge of the previously developed expert system to support clothing design process. In order to achieve the purpose of the research several subtasks were solved. Firstly, the collection of the transformable clothing was designed, and the method to analyse images was selected. The data set of images of transformable clothing was formed and prepared to be included into the database of the expert system, which is designed to select the clothing with certain emotional impression.
\end{abstract}

Keywords: transformable clothing; Kansei; semantic differential; expert system.

\section{INTRODUCTION}

Many consumers are contented with the fast fashion styles, abundant choices, and affordable price. However, other consumers and environmental advocates began to question about this fast fashion system, including the problems of overconsumption and disposable clothing. As a result, many fashion practitioners and scholars have been developing different strategies and methods to minimise the fabric waste, and prolong the product lifespan through innovative design [1].

The awareness of sustainability is increasing in diverse industries, including fashion industry. In order to reduce the harmfulness to the world, fashion industry is seeking appropriate ways to be more sustainable. Transformable clothing is one of the appropriate alternatives to reduce consumption and reinforce consumers to engage in sustainable lifestyle. Transformable design is an emerging design method which growing to be a fashion trend, and hence more fashion brands and designers are willing to invent transformable design. Thus, transformable fashion is very popular nowadays.

Researchers distinguish different types of clothes transformation: "detaching - attaching", "stretching - contracting", "regulating - fixing", "folding - unfolding", "showing - hiding", "overlapping (laying in) ", "replacing", "orienting", "rearranging", "segmentation", "overturning" $[2,3]$. Transformation process might refer to one of the mentioned types of transformation as well as to several of them that are used simultaneously. Any of the aforementioned types of transformation provides the ability for the transformable garment to change its function. Additionally, most of them change their function by the way of attaching or detaching some elements.

Transformable fashion can be basically defined as a garment that can be comfortably worn in multiple ways. Therefore, the impression from such clothes depends on a way they are worn. The main objective of this study is to evaluate the emotional component of the transformable clothing by using semantic differential.

\section{IRTTIE Vol. 6, No. 3, 2018 ISSN 1314-8788 (print), ISSN 1314-8796 (online), doi: 10.15547/artte.2018.03.006}




\section{ARTTLY $Y$}

Ipplied Researlohes in Technics, Technologies ind Bductation

Journal of the Faculty of Technics and Technologies, Trakia University https://sites.google.com/a/trakia-uni.bg/artte/

\section{METHODS}

Emotional design is extremely versatile trend that originated in Japan just three decades ago. In the 1970s the concept of Kansei Engineering appeared. This derivation used to transmit quality satisfaction from using some object or subject. Therefore, there are objects in which much Kansei, and there are those, which have it smaller or even not at all.

In order to perform transformable garment style selection Kansei Engineering methodology is to be applied. Kansei Engineering (KE) as a term, eventually transformed into "emotional design". KE develops methods of implementation of perceptual and emotional qualities into product design. The emotions which are caused by outfits play an important role in enhancing the aesthetic quality of clothing.

The basic method of image analysis in KE is the method of semantic differential (SD) in the classification of Kansei attributes [4]. SD is the method of psycholinguistics, which is a combination of scaling procedures and method of controlled associations [5].

This method allows to model semantic space that shows the relationship between the samples of products and meanings of words - adjectives that describe the impression from the products.

With the help of the bipolar scales of semantic differential the description of artistic and design solutions of clothes in the form of psychographic profiles was made [6]. Each profile is a list of the average meanings of the estimated coefficients of semantic differential. Identification of visual images of clothes models by the impressions that they produce was made by cluster analysis. As a result, clothes' models that have roughly the same psychographic profiles present each of clusters that are received. Combination of characteristics, which define each of clusters, are used in forming the productive model of expert system (ES) to support clothing design process that is developed and described in [7].

\section{EXPERIMENTAL}

Analysis of the emotional component of the garment on the basis of SD is achieved through cluster analysis. Such an approach identify models of transformable clothes based on perception and emotional needs of the consumer.

Cluster analysis is a process of multidimensional grouping of objects. In this case it is grouping of clothes depending on the impressions that they cause in consumer.

Using cluster analysis involves the constant action sequences, regardless of the subject of research. Accordingly, the sequence in this case is as follows:

- sampling selection for cluster - set of transformable garments images;

- determining the set of variables, which will be evaluated in the sample objects - a pair of Kansei words of SD;

- calculation of the degree of similarity between the objects;

- using cluster analysis method for grouping similar objects;

- results validation of cluster solutions.

The set of transformable clothes is presented in the Table 1. There are five transformable garment styles (models), which one of them is to be gathered from the set of parts. The garment parts are different by colours and texture. Thus, the variant of the model is the combination of these parts. Therefore, each model of transformable clothes might be presented by several variants (Table 1). Additionally, in the current research the factor of the colour brightness was taken into account. That is why the nominal number of the model under consideration consists of three numbers, the first of which represents the transformable garment style, the second reflects the variant of garment parts combination and the third number represent the colour brightness by scale from 1 to 3 , where 1 is soft and 3 is bright.

IRTTIE Vol. 6, No. 3, 2018 ISSN 1314-8788 (print), ISSN 1314-8796 (online), doi: 10.15547/artte.2018.03.006 


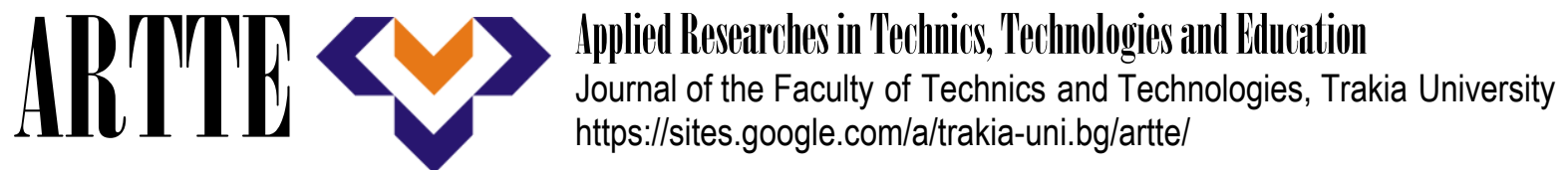

Table 1. Clothes models

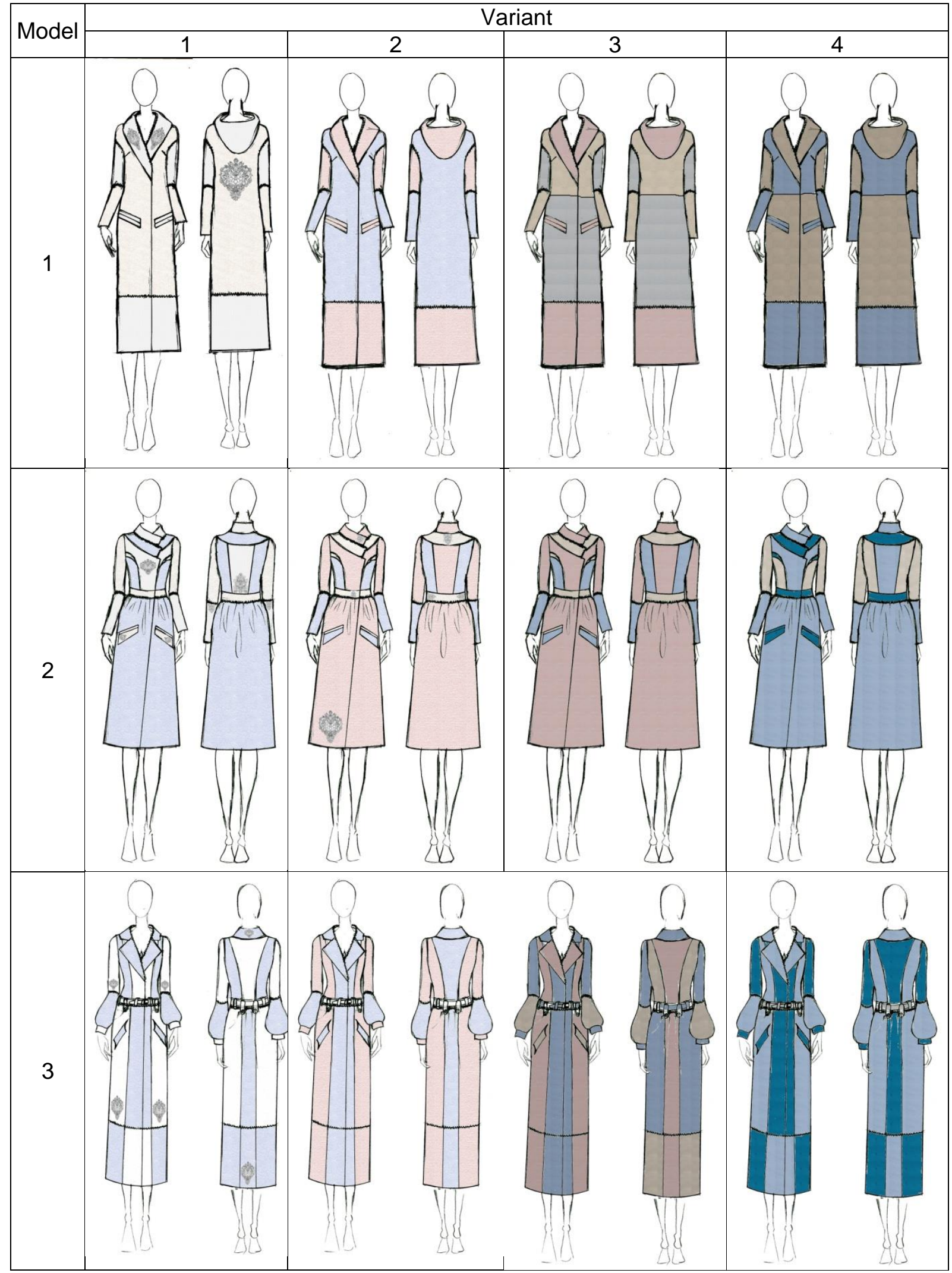

IRTTIE Vol. 6, No. 3, 2018 ISSN 1314-8788 (print), ISSN 1314-8796 (online), doi: 10.15547/artte.2018.03.006 


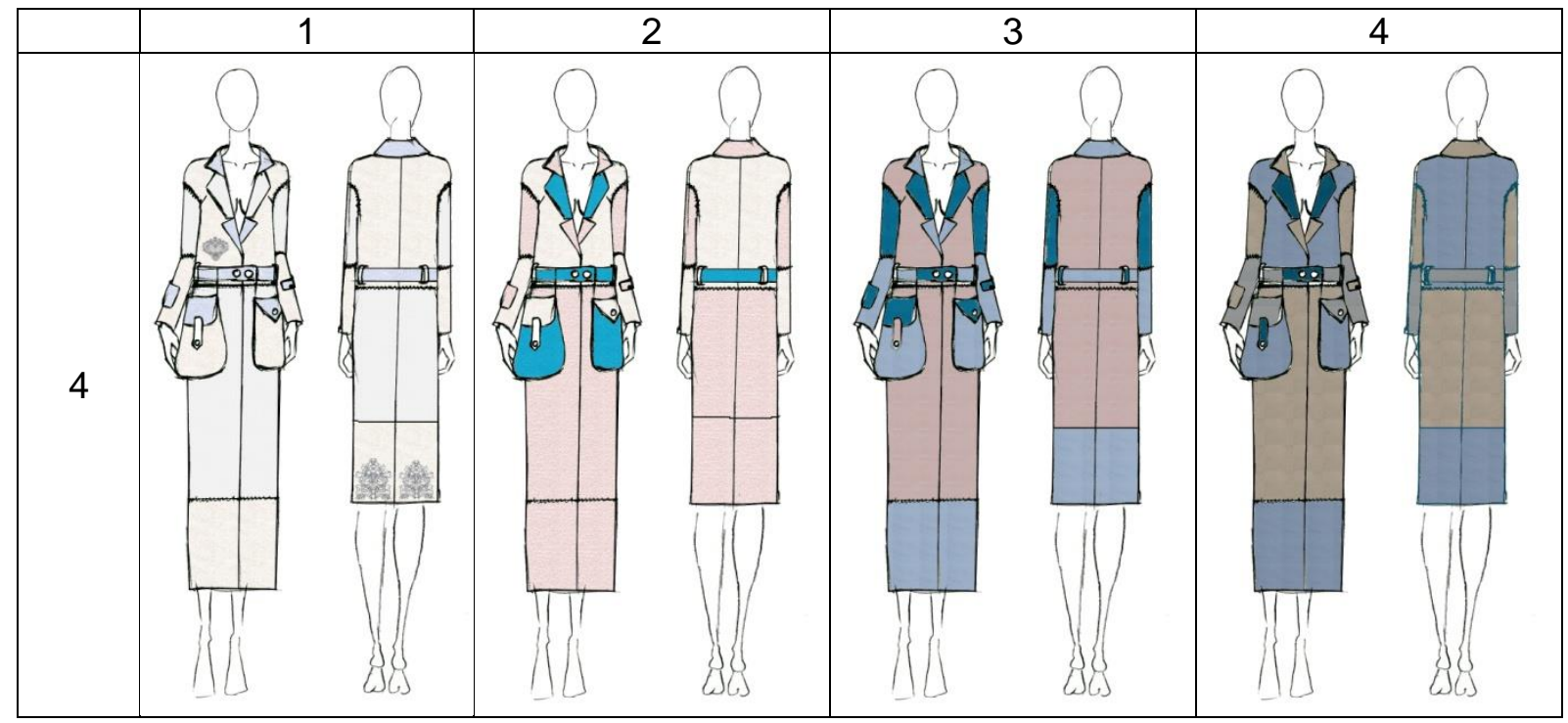

Cluster analysis can be used only when the following requirements are observed: performance should not correlate with each other, they should be dimensionless, their distribution should be close to normal, they must meet the requirement of 'stability', by which we mean no effect on their value random factors; in addition, the samples must be homogeneous and do not contain 'emissions'.

In order to implement these conditions it is necessary to conduct a preliminary factor analysis. These requirements are automatically met by the procedure of a factorial design. The factor analysis was performed during previously conducted research [7, 8]. Initial data for factor analysis were the profiles of each of the fashion models, presented as a series of average figures of evaluation ratios. As the results of the factor analysis, six factors (components) were highlighted, which can combine all pairs of words of SD that reflect consumer's impressions from clothes. That is why the number of descriptors of SD that must be considered for the transformable clothes in current research is restricted to six pairs of words. To facilitate communication between the man and the ES, which concept is being developed, for the design of system dialogues names of factors are presented by the most important components of each consistent. The names are as follows: $S A$ (SymmetryAsymmetry), BS (Bright-Soft), CS (Casual clothes - Smart clothes), TN (Transparent texture - Non-transparent texture), FM (Folk clothes - Modern clothes), TdTu (Trapesoid shape (long base down) - Trapezoid shape (long base up)).

Group of experts assessed the transformable garments. The result of the assessment was presented as a psychographic profile of each transformable outfit. The profiles reflect the average amounts of evaluation coefficients for six pairs of Kansei words.

Bipolar adjectival pairs of Kansei words - simple, economical tools that make it possible to get some data based on a subjective understanding of connotative meanings of words by people. These tools include a few scales put horizontally on a form (questionnaire). Each scale has seven gradations that are expressed numerically $(-3,-2,-1,0,+1,+2,+3)$ or verbally (hard, medium, low, can, low, medium, hard) (Figure 1). 

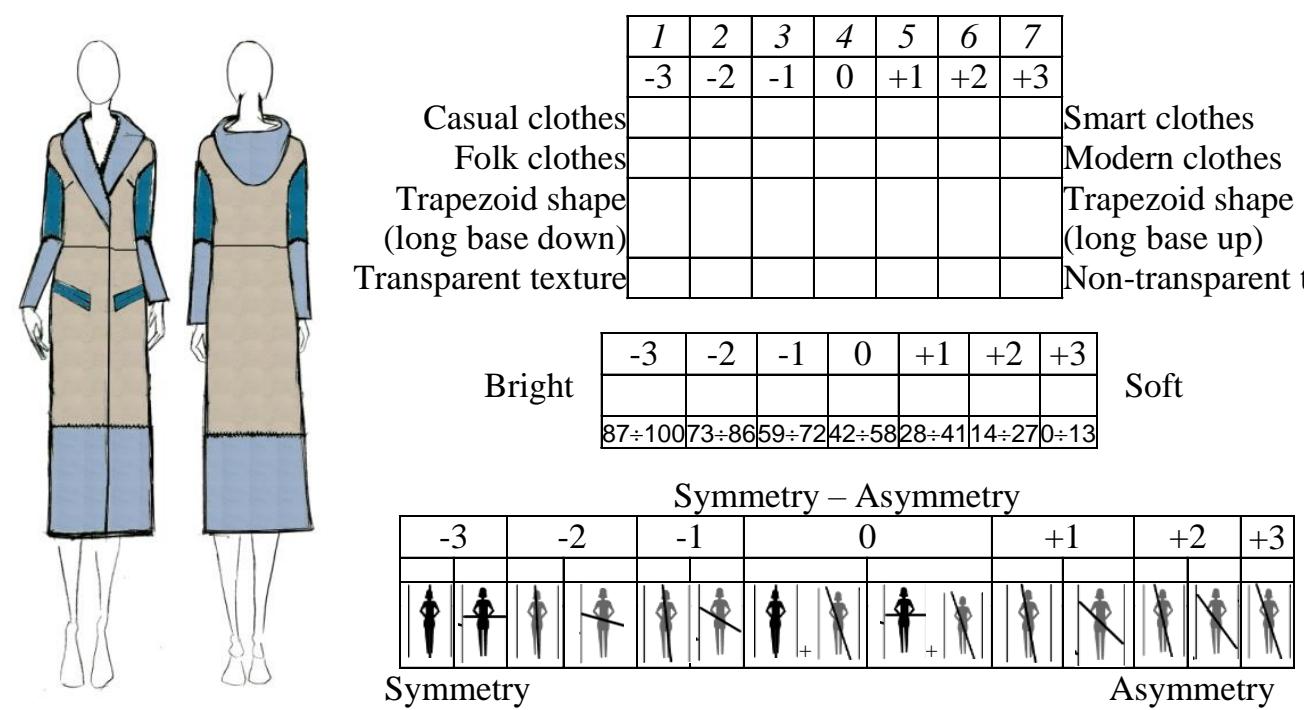

Figure 1. Example of the questionnaire

As a result of the cluster analysis 67 conventional groups were selected (Table 2). Groups are formed of models with closest bonds that differ less than $20 \%$.

Table 2. Interpretation of clusters (fragment)

\begin{tabular}{|c|c|c|c|c|c|c|c|}
\hline \multirow{2}{*}{ Cluster } & $f_{1}$ & $f_{2}$ & $f_{3}$ & $f_{4}$ & $f_{5}$ & $f_{6}$ & Clothes models \\
\hline 1 & 2 & 3 & 4 & 5 & 6 & 7 & 8 \\
\hline 26 & -3 & 1 & -1 & 2 & 1 & -2 & 143 \\
\hline 27 & -2 & 0 & -2 & 2 & 1 & -1 & 233 \\
\hline 28 & -2 & 0 & -1 & 0 & 0 & -2 & $132 ; 152$ \\
\hline 29 & -2 & 0 & -1 & 1 & 1 & -2 & 343 \\
\hline 30 & -2 & 0 & -1 & 2 & 1 & -2 & $163 ; 313 ; 323 ; 333 ; 363$ \\
\hline 31 & -2 & 1 & -1 & 2 & 1 & -2 & $13 ; 153 ; 13 ; 193$ \\
\hline 32 & -2 & 0 & -1 & 2 & 1 & -1 & $223 ; 353 ; 453 ; 473 ; 483$ \\
\hline 33 & -2 & 0 & -1 & 2 & 1 & 0 & $253 ; 263 ; 273 ; 283$ \\
\hline 34 & -2 & 0 & 0 & 2 & 1 & 0 & 423 \\
\hline 35 & -2 & 1 & -3 & 2 & 1 & -2 & 183 \\
\hline 36 & -2 & 1 & -1 & 1 & 0 & -2 & $123 ; 133$ \\
\hline 37 & -2 & 1 & -1 & 1 & 1 & -1 & 463 \\
\hline 38 & -2 & 1 & -1 & 2 & 1 & -1 & $213 ; 413 ; 433 ; 493$ \\
\hline 39 & -2 & 1 & -1 & 2 & 1 & 0 & 243 \\
\hline$\ldots$ & $\ldots$ & $\ldots$ & $\ldots$ & $\ldots$ & $\ldots$ & $\ldots$ & $\ldots \ldots$ \\
\hline 44 & -1 & 1 & -1 & 0 & 0 & -2 & $142 ; 172$ \\
\hline$\ldots$ & $\ldots$ & $\ldots$ & $\ldots$ & $\ldots$ & $\ldots$ & $\ldots$ & $\ldots$ \\
\hline 52 & 0 & 0 & -2 & 0 & 0 & 0 & $242 ; 272$ \\
\hline 1 & 2 & 3 & 4 & 5 & 6 & 7 & 8 \\
\hline 53 & 0 & 0 & -1 & 0 & 0 & -1 & $212 ; 222$ \\
\hline$\ldots$ & $\ldots$ & $\ldots$ & $\ldots$ & $\ldots$ & $\ldots$ & $\ldots$ & $\ldots$ \\
\hline 69 & 1 & 1 & -1 & -2 & -2 & -2 & $141 ; 171$ \\
\hline$\ldots$ & $\ldots$ & $\ldots$ & $\ldots$ & $\ldots$ & $\ldots$ & $\ldots$ & $\ldots$ \\
\hline 82 & 2 & 0 & -2 & -2 & -1 & 0 & $231 ; 241$ \\
\hline 83 & 2 & 0 & -2 & -3 & -2 & 0 & $\ldots$ \\
\hline$\ldots$ & $\ldots$ & $\ldots$ & $\ldots$ & $\ldots$ & $\ldots$ & $\ldots$ & $\ldots$ \\
\hline 92 & 2 & 0 & 0 & -2 & -2 & -1 & 431 \\
\hline
\end{tabular}

IRTTIE Vol. 6, No. 3, 2018 ISSN 1314-8788 (print), ISSN 1314-8796 (online), doi: 10.15547/artte.2018.03.006 


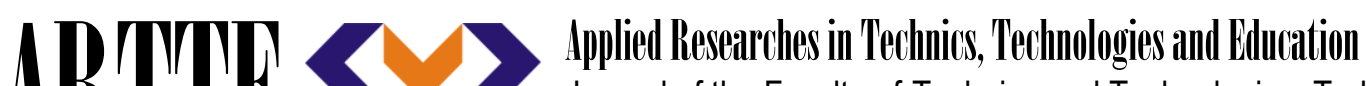 Journal of the Faculty of Technics and Technologies, Trakia University https://sites.google.com/a/trakia-uni.bg/artte/}

According to the results of cluster analysis, we may form a productive ES models for choosing clothes considering the wishes (opinions) of a consumer. ES model of production presupposes knowledge of the relationship between the concepts. The relationship between the concepts is presented in the form of ordered sequences $C l_{i}=\left(f_{1 i}, f_{2 i}, f_{3 i}, f_{4 i}, f_{5 i}, f_{6 i}\right)$ and $\mathrm{M}=$ $\left(\mathrm{Cl}_{i}\right.$, colour), where $\mathrm{i}$ is a model number.

The factors that have a high degree of influence in clusters, cluster analysis is performed with k-mediums, the results of which are presented in Table 2, where the final cluster centres are sorted according to the figure of the first factor. None of them is similar to the one of 25 previously selected clusters [7]. The results of the current research are to improve the developed ES. For that reason the clusters are numbered from 26 to 92 (Table 2).

\section{RESULTS}

As it is shown in the Table 2, the very same transformable clothes make different impressions depending on their parts combinations and color characteristics. While there were only five basic models of clothes assessed, the number of clusters or in other words the number of different impressions is a lot greater. That is why it is advisable to use the expert system (ES) to select a transformable garment with certain emotional impression.

The ES knowledge base for solving the subtasks of choosing the models of readymade garments based on KE methodology in the shell "Rapana" [9] has been developed and described in [7]. The shell 'Rapana' is distributed free of charge via the web site (http://esrapana.narod.ru/). The knowledge base provides dialogue as a series of questions by the system and answers by the user. This system can be used for the selection of readymade garments (eg. in the shops, including online stores) and to select a prototype to develop new model of clothing that meets the wishes of the consumer.

The obtained results are to be used as an improvement of the mentioned expert system. Thus, the information in the Table 2 is a productive model of the ES. The way of decisionmaking is the same as it is described in [7]. The major changes are mostly in the image set that supports the productive model of ES. The set is to include the images of transformable clothes that is under assessment.

\section{CONCLUSIONS}

The main objective of the study that is to evaluate the emotional component of the transformable clothing by using semantic differential was achieved by solving several subtasks.

Firstly, the collection of the transformable clothing was designed, and the method to analyse images was selected. The data set of images of transformable clothing was formed and prepared to be included into the database of the expert system, which is designed to select the clothing with certain emotional impression.

\section{ACKNOWLEDGMENT}

The results shown in the paper resulted from the Scientific Project "Development of the principles of heuristic clothing design to develop a prototype of the expert system for rapid change in production of clothes", Grant of the Ministry of Education and Science of Ukraine № $0117 \mathrm{U} 003889$. 


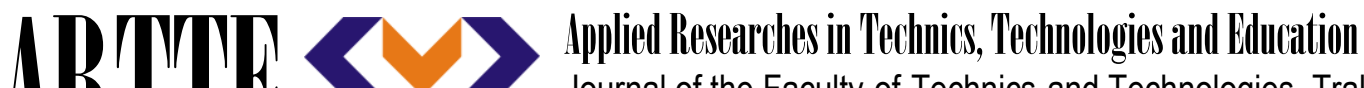 Journal of the Faculty of Technics and Technologies, Trakia University https://sites.google.com/a/trakia-uni.bg/artte/}

\section{REFERENCES}

[1] Rahman O., \& Gong M. (2016). Sustainable practices and transformable fashion design Chinese professional and consumer perspectives. International Journal of Fashion Design, Technology and Education, Vol. 9, No. 3, (2016).

[2] Shamuhitdinova L., Chursina V., \& Kamilova K. (2002). Analysis of historical prototypes of the methods of morphological clothing transformation, Sanat, No. 3, (2002).

[3] Beskorovaynaya G.P., \& Kurenova S.V. (2000). Design of children's clothing: Study guide, Craftsmanship, Moscow, (2000).

[4] Nagamachi, M. (2011). Kansei / Affective Engineering. Taylor \& Francis Group (2011).

[5] Osgood, C. E. (1968). Method and Theory in Experimental Psychology. Hardcover: Import, (1968).

[6] Kuleshova, S. G., \& Slavinska, A. L. (2015). Method of complex assessment of aesthetic quality in clothes design. Shalapko Y., Wyszkowska Z., Musial J., Paraska O, Study of problems in modern science: new technologies in engineering, advanced management, efficiency of social institutions. Monograph. Bydgoszcz: Poland, (2015), pp. 318-327.

[7] Kuleshova S. G., Zakharkevich O. V.,. Koshevko J. V. \& Ditkovska O. A. (2017). Development of expert system based on Kansei Engineering to support clothing design process. Vlakna a Textil, Vol. 4, (2017), pp. 30-41.

[8] Kurochka S., Sviruk L., Kuleshova S. \& Zakharkevich O. (2017). Method of analyzing images of clothes based on Kansei Engineering. International Conference on Technics, Technologies and Education ICTTE 2017, Yambol, Bulgaria, October 19-20st, (2017), pp. 92-99.

[9] Expert system "Rapana". [Online]. Retrieved 11. 16. 2016. Available: http://esrapana.narod.ru/. 